\title{
Inflation expectations quantification with fuzzy control system
}

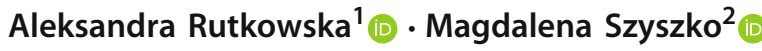

Published online: 19 February 2021

(c) The Author(s) 2021

\begin{abstract}
The inflation expectations of economic agents are one of the most important variables for central banks and monetary policy conduct, but such expectations are not directly observable. Consumers' expectations are examined in qualitative surveys. The key task is to transform consumers' survey responses into quantitative proxies of expectations. In this examination, we investigate an alternative method to quantify consumers' inflation expectations using fuzzy rule-based systems. In order to generate rules in the learning phase, we compare two methods: the Wang-Mendel method based on space partition and the subtractive clustering method. The learning data are information about past inflation and consumers' opinions as expressed in surveys. The system is built and tested on data from January 2002 to June 2019 for non-euro member states of the EU. The fuzzy rules-based system returns results that outperform a benchmark - the standard quantification procedure-in terms of correlation with a raw approximation of consumer expectations, which is survey balance statistics. The results are robust regarding the benchmark choice. We find the method more promising for economies with lower stability, as it gives more room for the training phase of the algorithm application.
\end{abstract}

Keywords Fuzzy control system · Inflation expectations · Clustering

\section{Introduction}

In this paper, we present a novel application of a fuzzy rules-based algorithm for the quantification of consumer expectations. Individuals' expectations are the subjective beliefs they hold about uncertain future economic outcomes (Pesaran and Weale 2006). Inflation expectations are a pivotal variable for central banks: modern monetary theory asserts that successful expectations management results in more effective monetary policy. Standard measures that approximate expectations-especially when consumers are involved-have been subject to criticism. Hence, we are motivated to discuss a methodological innovation that avoids the majority of objections against standard methods.

Communicated by Vladik Kreinovich.

Aleksandra Rutkowska

aleksandra.rutkowska@ue.poznan.pl

Magdalena Szyszko

magdalena.szyszko@wsb.poznan.pl

1 Poznan University of Economics and Business, Poznan, Poland

2 WSB University in Poznan, Poznan, Poland
We use monthly data regarding consumer expectations from Business and Consumer Surveys in nine EU member states from 2002 to mid-2019. The survey is qualitative: consumers express their directional opinions about price evolution. Thus, we apply a fuzzy rules-based system to quantify these data. The Wang-Mendel method, which is based on space partition, and the subtractive clustering method are applied to discover learning rules concerning inflation history and the structure of consumers' responses as expressed in the surveys.

Our research procedure comprises a fuzzy rules-based results comparison with the outcomes of a standard quantification procedure. We juxtapose our results with two time series that we generated with the application of two versions of the standard probabilistic quantification method. Our method outperforms them both in terms of correlations with balance statistics that express unbiased consumer opinions. The results are more favourable for economies with a higher degree of inflation fluctuations, as more volatility gives more room for the learning algorithm. This finding holds when we apply an alternative benchmark.

The remainder of this paper is organised as follows: Sect. 2 presents the economic background of the problem we address. Section 3 describes the application of the 
fuzzy rules-based system to this problem. Section 4 provides empirical examination results with a robustness check and economic interpretation. Finally, the last section concludes the article.

\section{Problem statements and assumptions}

Expectations are not directly observable economic variable, and economists search for approximations of expectations, often deriving them from economic models solved under the assumption of the expectations formation process. However, as this assumption could be questioned because economists are unsure of how expectations are actually formed, direct proxies of expectations are preferred. Expected inflation is embedded in the term structure of interest rates. Consequently, the observation of financial asset prices can serve as a proxy for expectations. Alternatively, the direct proxies of expectations are survey-based. Surveys conducted among professionals, business people, and consumers are the only measure for estimating consumers' expectations. In this study, we focus on the way in which consumers' surveys results are transformed into proxies of expectations in an attempt to identify an innovative method for quantifying consumers' expectations that avoids the objections that are typically raised towards standard methods and respects limitations regarding individuals' abilities to acquire and process economic information.

\subsection{Survey}

Central banks refrain from examining consumers' inflation expectations in a quantitative manner. The results of quantitative surveys are volatile, register high dispersion, and do not reflect the actual economic situation. The Czech National Bank explained its choice to abandon quantitative surveys by stating that recently, the values of this indicator [expectations proxy] have been highly volatile and have failed to give a clear picture of inflation expectations (Czech National Bank 2007). This perspective reflects the usefulness of quantitative surveys. The low degree of economic knowledge and awareness of ongoing and future situations leave room for presumptions; consumers' declarations about the future are typically vague. Qualitative surveys designed to assess economic agents' sentiments are broadly applied by national entities to estimate consumer expectations. EU member states are obliged to run surveys with a common methodology, such as Business and Consumers Surveys (BCS), under the auspices of the European Commission (European Commision 2020). BCS contain the following question about perceived inflation (Q5):
How do you think that consumer prices have developed over the last 12 months? They have...: risen a lot, risen moderately, risen slightly, stayed about the same, fallen, don't know.

Because inflation perception could be used to proxy expected inflation rates (as a scaling factor), we present both questions. The most relevant question for consumer expectations is Q6:

In comparison with the past 12 months, how do you expect that consumer prices will develop in the next 12 months? They will...: increase more rapidly, increase at the same rate, increase at a slower rate, stay about the same, fall, don't know.

Business and Consumers Surveys are conducted monthly in EU member states and official candidate states. Similar surveys are employed in numerous countries beyond the EU to investigate consumer expectations.

\subsection{Standard quantification methods}

The aggregate results of the Business and Consumers Surveys - the percentages of responses to the survey's questions - are publicised and may be used to obtain a direct, quantitative proxy of inflation expectations. Such proxies are typically valued even if some examinations are based directly on survey responses (Acedański and Włodarczyk 2016). There are two acknowledged and commonly applied methods of expectations quantification: probabilistic methods and regression methods (Table 1). The set-ups and reliability of both methods have been criticised. Nonetheless, there is no other broadly accepted alternative for quantification.

Economic and econometric objections towards these methods are broadly discussed in the literature. Objections rooted in economics take issue with the low reliability of the results. Lahiri and Zhao (2015) provided findings that suggest the lack of correlation of the results of quantitative surveys and quantified surveys, referring to the University of Michigan's Survey of Consumers, which provides both qualitative and quantitative results. The other study suggests a more realistic version of the probability method, which is assumptions-free, and acknowledges that consumers are governed by the official central bank inflation targeting policy in discriminating between moderate and extreme inflation tendencies (Lolić and Sorić 2018). Unrealistic assumptions are the most criticised element of the existing methods. An earlier study by Henzel and Wollmershäuser (2005) provided a modification that relaxed the unbiasedness assumption regarding time series in question for Carlson and Parkin's procedure.

Existing methods of quantification hardly compromise economic and econometric requirements. The set of assumption needed for quantification is rarely reflected in time series properties. Numerous extensions complicate econo- 
Table 1 Expectations quantification methods

\begin{tabular}{lll}
\hline & Probabilistic methods & Regression methods \\
\hline Author(s) & Theil (1952);Carlson and Parkin (1975) & Anderson (1952); Pesaran (1984) \\
Idea & Individual percentages of respondents are expressed & It is assumed that the same relationship holds between \\
& in terms of the probabilities of future inflation being in & respondents' qualitative opinions concerning future \\
& certain intervals. Additionally, sensitivity intervals are & price changes and expected inflation. Thus, inflation \\
& applied for (1) respondents declaring that prices will & perception is a yardstick for the quantification of \\
& be stable (they do not necessarily mean that future & respondents' expectations. Coefficients derived from \\
& inflation will be exactly equal to zero) and for (2) & Q5 answers are used together with Q6 answers to \\
& respondents reporting that prices will increase at the & reveal expected inflation
\end{tabular}

Assumptions

Versions

Extensions

Critique same rate (they do not necessarily mean that future inflation will be exactly equal to zero). Solving a set of equations returns the expected inflation rate, its standard deviation, and sensitivity intervals

The original Carlson and Parkin approach assumes the unbiasedness of inflation expectations and their normal distribution. Extensions remove the former and allow for the modifications of the latter. There is a need for an assumption about the scaling factor to which respondents refer while answering the survey's questions

Regarding the scaling factor: objectified (actual inflation rate serves as a scaling factor) and subjectified (perceived inflation). Regarding the distribution: uniform, logistic, t-distributions, triangular distribution

The most commonly applied extension is that of Batchelor and Orr (1988). It adjusts the probabilistic method to polychotomous (five-question) surveys, which are currently a standard tool for examining expectations, broadly expanding the literature regarding the method and aiming at limiting method's shortcomings and adjusting it to modern economic conditions

Unrealistic assumptions and difficulties regarding the empirical application of the method; empirical distributions do not mimic theoretical distributions of variables; accuracy of quantified expectations
Unbiasedness of inflation perception. Additional assumptions differ across the models applied. No assumption about distribution needed
The primary version of the procedure was presented by Anderson (1952). Coefficients of Anderson's model do not change over time even if the inflation dynamic changes. This is the most important objection towards the simplest method

Models by Pesaran (1984), Smith and McAleer (1995) allowed for different solutions regarding general price level changes. Pesaran introduced asymmetrical relation of perceived inflation and expected price change. Smith and McAleer presented a model with timevarying parameters. Simmons and Weiserbs (1992) model was designed to work for the polychotomous survey

Problems related to models' estimations including standard econometric problems as the choice of estimator. Designed mostly for use in three-question surveys. Modern surveys of expectations are extended to five questions metric procedures while moving the economic background of the method towards the economic reality. Even if timevarying parameters set-ups better reflect consumers' economic behaviour, they are not typically applied in empirical examinations and fail to meet econometric proprieties.

The results of surveys could be used to find a fuzzy rulesbased method of quantification that outperforms existing quantification procedures. We use the structure of responses to the survey's questions to propose a method of quantification that does not replicate the shortcomings of the standard quantification methods that we discussed above.

\section{Fuzzy system approach to expectation quantification}

Fuzzy control incorporates ambiguous human logic into computer programs. It suits problems that cannot be easily represented by mathematical models and systems for which there are very weak standards models. Fuzzy systems are also well suited to economic intuition regarding the ways in which consumers form and express their expectations. Regardless of the theoretical premises assuming their ability to be rational in Muth's sense (Muth 1961), they are far from rationality. Their assessment of future inflation can be described in terms of uncertainty, not risk. The ambiguity of human logic perfectly matches their behaviour. Additionally, the important advantage of fuzzy systems' application is their lack of assumptions regarding the time series properties. 
Fig. 1 Fuzzy Control System scheme

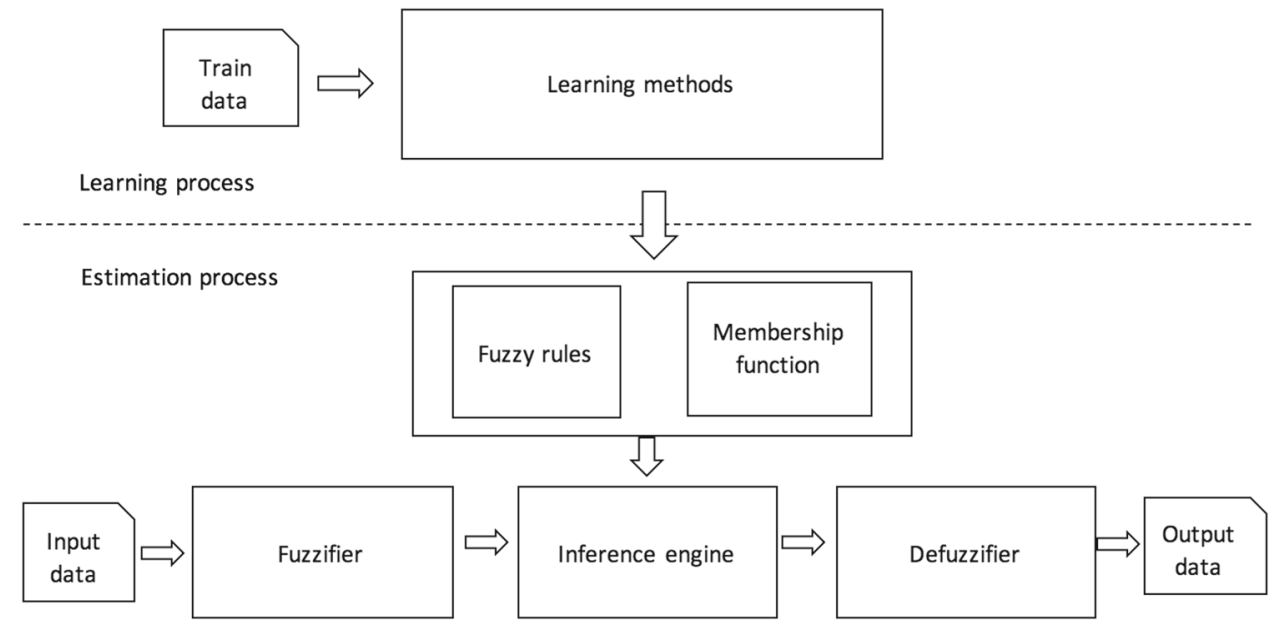

Such assumptions are often volatile and make econometric methods hard to apply in reality.

\subsection{Fuzzy control system}

Fuzzy control was initiated by Mamdani and Assilian (1975) in 1974 stimulated by the Zadeh's two seminal papers (Zadeh 1973, 1996), in which Zadeh presented modelling based on fuzzy rules IF-THEN (Nguyen et al. 2019). Regardless of the fuzzy control system (FLC) type, the operation diagram is as shown in Figure 1. In the case under examination, although the general rules for changing the results of the type surveys are known f.e. IF the number of responses that the prices have risen a lot is large AND the number of responses that the prices have fallen is small THEN the expected inflation is high. Such rules are too general, and the meaning of the word 'small' will vary significantly depending on the country and even the time. Thus, we must consider the learning system. The generation of regulations will be based on the perception of inflation by the respondents checked by the question Q5 described in Sect. 2.1. Due to the small available learning sample, the use of methods using neural networks or genetic methods is inadvisable. Two standards will be used in this study: the Wang-Mendel method (WM Wang and Mendel 1992) and subtractive clustering (SBC Chiu 1996), which is an extension of Yager and Filev's mountain method (Yager and Filev 1994).

The WM method can be described by the following steps:

1. Set a fuzzy partition of the input variable space. Each domain interval $k, k=1 \ldots m$, is divided into $2 n+1$ regions ( $n$ is a natural number), where the centre of each corresponding membership function lies in the centre of the region, and the extrema lie at the centre of the neighbouring regions.

2. Generate a preliminary linguistic rule set. For each inputoutput pair, a rule is generated by:
- Compute the matching degree of pair to different fuzzy regions using a conjunction operator.

- Assign the pair to the fuzzy region with the highest membership degree.

- Generate a rule.

- Assign an importance degree to each rule.

3. Remove conflicting rules.

4. Create a final rule base with the rules of highest importance

The interest in this method resides in its simplicity and its straightforward approach, which at the same time has proven capabilities to provide reasonably good performance.

SBC (Chiu 1996) considers each data point as a potential cluster centre by determining the potential of a data point as a function of its distances to all the other data points. A data point has a high potential value if that data point has many nearby neighbours. The highest potential is chosen as the cluster centre, and the potential of each data point is updated. The process of determining new clusters and updating potentials is repeated until the remaining potential of all data points falls below some fraction of the potential of the first cluster centre.

\subsection{Expectation quantification}

Let $x=\left[x_{P P}, x_{P}, x_{E}, x_{M}, x_{M M}, x_{i n f}\right]$ be the vector of training data, where the first five are percentages of answers to Q5 (How do you think that consumer prices have developed over the last 12 months? They have...), as follows:

$x_{P P}$ risen a lot;

$x_{P}$ risen moderately;

$x_{E}$ risen slightly;

$x_{M}$ stayed about the same;

$x_{M M}$ fallen; 
Table 2 Descriptive statistics of inflation in study period

\begin{tabular}{lllllllllc}
\hline Country & Mean & Sd & Median & Trimmed & Mad & Min & Max & Skew & Kurtosis \\
\hline BG & 3.75 & 4.16 & 3.10 & 3.29 & 3.26 & -2.22 & 26.80 & 1.88 & 6.86 \\
HR & 1.96 & 1.81 & 2.00 & 1.90 & 1.78 & -1.30 & 6.30 & 0.26 & -0.4 \\
CZ & 2.04 & 1.40 & 2.10 & 1.88 & 1.04 & 0.00 & 6.6 & 1.00 & 1.18 \\
DK & 1.59 & 0.92 & 1.50 & 1.55 & 1.19 & -0.10 & 4.40 & 0.38 & -0.47 \\
HU & 3.91 & 2.56 & 3.75 & 3.91 & 2.74 & -1.40 & 9.00 & -0.01 & -0.85 \\
PL & 1.95 & 1.65 & 1.85 & 1.98 & 1.85 & -1.6 & 5 & -0.09 & -0.9 \\
RO & 6.28 & 5.93 & 4.95 & 5.55 & 4.67 & -3.46 & 28.6 & 1.31 & 2.07 \\
SE & 1.25 & 1.21 & 1.30 & 1.21 & 1.19 & -1.9 & 4.4 & 0.2 & -0.24 \\
UK & 2.17 & 1.12 & 2.10 & 2.14 & 1.04 & -0.10 & 5.20 & 0.26 & 0.04 \\
\hline
\end{tabular}

and $x_{\text {inf }}$ is measure of inflation-we apply: current inflation, and alternatively inflation delayed by two periods. Next, we set the range, minimum and maximum value, for all variables, although by definition, variables are the percentage of responses to a survey question, their value is in the range of 0 1. In practice, there is never such unanimity that any values assume 1 or values very close to it. The range of expectations is even more difficult to determine. Next, the method of rule-based function generation is chosen as well as additional parameters such as the number of labels (fuzzy sets) for each variable. Then, in the learning phase, rules are generated using the WM method and SBC algorithms. Afterwards, the expectation is quantified based on answers to question Q6 By comparison with the past 12 months, how do you expect that consumer prices will develop in the next 12 months? They will..., the answers for which have a similar structure as those for question Q5. Question Q5 and its structure of responses are used in an analogous way, as occurring when regression methods are applied: it constitutes the pattern for finding (learning) regarding expected inflation.

\section{Empirical study}

\subsection{Data and methods}

Our sample covers nine non-euro area EU member states: Bulgaria, Croatia, Czechia, Denmark, Hungary, Poland, Romania, Sweden, and the UK ${ }^{1}$. We chose these countries in consideration of sample diversification: the sample includes transitioning and advanced economies, countries that have recently experienced high and volatile inflation rates, and economies with a high degree of economic stabilisation. Our sample has a common monetary policy framework and is covered by BCS. This sample makes it possible to test a new approach for cases in which standard methods return

\footnotetext{
1 The UK was an EU member state for the entire period covered by our examination.
}

unreliable results. Standard procedures return weak or statistically insignificant correlations for Danish consumers' expectations and their balance statistics.

The whole sample covers monthly data from January 2002-June 2019 (210 observations). We start our sample in 2002 because prior to this year, divergences in our sample regarding monetary policy were much more significant. This is a large sample, when the macro-data are considered. However, we are aware that in the case of generating automatic decision-making rules, it is not a large amount of data, and some methods (e.g. those based on neural networks) cannot be used. To illustrate the nature of the sample, descriptive statistics on inflation from the period under examination in specific countries are presented in Table 2.

Countries from our sample exhibit differences in inflation levels and volatility. Developed economies performed better in terms of inflation stabilisation. Bulgaria and Romania represent the other extreme. As the success of fuzzy system-based rules application for expectations quantification depends-to some extent-on inflation volatility, we refer to inflation properties while interpreting the results.

For the learning set, next to current inflation, we place inflation delayed by two periods because it is the last published inflation known to consumers. In the empirical study, we set expectations using fuzzy systems in the following cases:

- WM_inf: rules generated by WM methods used as learning set: answers for Q5 and current inflation;

- WM_inf2: rules generated by WM methods used as learning set: answers for Q5 and inflation delayed by two periods;

- SBC_inf: rules generated by SBC methods used as learning set: answers for Q5 and current inflation;

- SBC_inf2: rules generated by SBC methods used as learning set: answers for Q5 and inflation delayed by two periods-inflation known in the current period from official publication sources; 
We test the WM methods on different labels number: 3 and 5 . Due to the large differences in the characteristics of the data of each country, the range of data necessary for the application of the WM method was determined for each country separately. Ranges for particular countries are presented in "Appendix A" and set using the following rules:

- range 1: by min and max value from survey data and inflation data

- range 2: by min and max value from survey data and inflation data including heuristics resulting from data from Q5 and inflation is extended by 5-10 pp. or by 1-3 pp. dependent on the stability of inflation in the country and rounding to integer values.

To examine the performance of the system, in the absence of any actual expectation values, the Pearson correlation coefficient (eq.(1)) was calculated with the balance statistics from question Q6. Balance statistics (eq. (2)) represent the raw and aggregate representation of consumers' opinions about expected inflation rates. They cannot be interpreted in terms of inflation expectations. However, balance statistics are unbiased by any assumption relating to consumers' opinions about inflation in the forthcoming periods.

$$
\begin{aligned}
R & =\frac{\sum_{i=1}^{n}\left(x_{i}-\bar{x}\right)\left(y_{i}-\bar{y}\right)}{\sqrt{\sum_{i=1}^{n}\left(x_{i}-\bar{x}\right)} \sqrt{\sum_{i=1}^{n}\left(y_{i}-\bar{y}\right)}} \\
B S & =\left(x_{P P}+\frac{1}{2} x_{P}\right)-\left(\frac{1}{2} x_{M}+x_{M M}\right)
\end{aligned}
$$

where $x_{P P}$ represents the percentage of the 'most positive answer' (e.g. for Q5: 'prices have risen a lot' and for Q6: 'prices will increase more rapidly'), $x_{P}$ the percentage of 'positive' option, $x_{M}$ - the percentage of respondents having chosen the option 'negative', and $x_{M M}$-the option 'very negative'.

The higher the correlation coefficient, the better. It is not possible to verify directly whether fuzzy system-based rules outperform any other quantified proxy of expectations. We do this indirectly by using a benchmark method and identifying the correlation between the balance statistics of expectations and our benchmark. We distinguish two benchmarks: subjectified $\left(C P_{s u b}\right)$ and objectified $\left(C P_{o b j \_i n f}\right)$ (Carlson and Parkin 1975) benchmarks. They are the most commonly applied quantification procedures for empirical examinations and central banks analyses. We distinguish:

- subjectified $\left(C P_{s u b}\right)$ version of quantification — set using a two-step procedure: Q5 structure of answers and a representation of 'normal' inflation are used to quantify the perceived inflation rate. The quantified perception of inflation is a scaling factor used to proxy expectations together with Q6 answers

- objectified $\left(C P_{o b j \_i n f}\right)$ version-using the official inflation statistics as a scaling factor.

Eventually, we juxtapose the correlation examination results and compare associations registered for fuzzy system-based rules and standard quantification procedures.

\subsection{Results}

Table 3 presents the results of the correlation. The bold cell indicates that the result is higher than both benchmarks, whereas the italic cell indicates that it is higher than one benchmark and underline font means statistically insignificant correlations. The benchmark correlation is presented in "Appendix B". In $65 \%$ of cases, we received a result with a higher correlation from at least one of the standard benchmarks. This percentage increases to $75 \%$ if we only consider results from range 2. Obviously, the SBC method performs much better than the WM method. In $83 \%$ of the cases, the correlation was higher than the benchmark. If the optimal settings are chosen, (the SBC and range 2 methods), in 18 out of 18 cases (in $89 \%$ of cases), the fuzzy rule-based method returns better results. Once we search for a rationale of the results, we refer to inflation volatility: countries with higher and more volatile inflation give more room for our learning mechanism to capture the behaviour of expectations. However, the mechanics of fuzzy rules-based systems needs to be compromised in consideration of the economic reality of consumers. Hungary proved to be a special case herehaving relatively volatile inflation, this economy registers a lower correlation of fuzzy system-based results in comparison with a standard benchmark. To some extent, we can present an alternative explanation for the Hungarian case: the Hungarian economy underwent a number of structural changes regarding monetary policy conduct, as well as institutional and political pressure. This could affect consumers' abilities to assess the situation. If the structure of respondents' answers to the survey was affected by economic shocks, the learning system had no chance to capture the rule properly. Results for the remaining economies did not diverge from our economic intuition, including the UK case. The UK inflation was kept stable, and Brexit offered a beyond-the-scale economic shock. However, fuzzy rules-based systems performed quite well here- - the strength of the correlation was not impressive, but it was outperformed the benchmark for the majority of cases. Quite the opposite situation occurred in the most stable economy in the sample-Sweden. The overall economic stability of Sweden could be reflected in the stable structure of responses to the survey's questions, which constrained the learning mechanism. 
Table 3 Correlation of inflation expectation and balance statistics

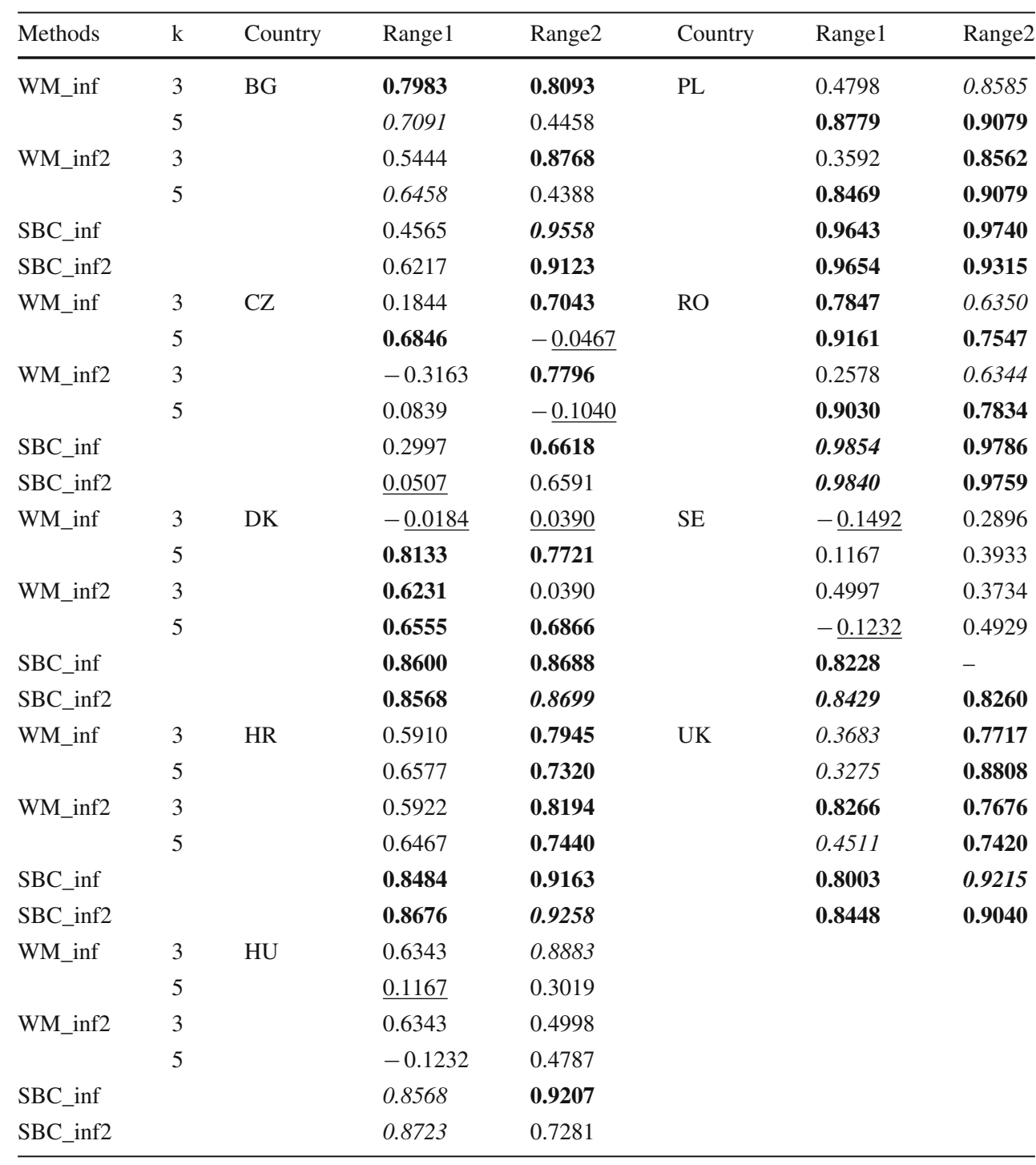

Underline results are statistically insignificant

\subsubsection{Robustness check}

We offer a multi-benchmark procedure as a robustness check of our results. Instead of the application of procedures chosen arbitrarily according to the criterion that they are "the most commonly applied' $\left(C P_{\text {sub }}\right)$ and $\left(C P_{o b j \_i n f}\right)$, we offer a time series quantified with the application of a different variation of a standard probabilistic method. Our procedure for choosing the benchmark consists in:

- quantification of expectations according to 35 different variations for each economy. We applied five different distributions and seven scaling factors for each distribution,

- identification of the best, individualised for each economy method according to two valuation criteria: (i) the strongest correlation with balance statistics and (ii) the best forecast accuracy according to Theil's coefficient of inequality.

We avoid a detailed explanation of the procedure as it relates to benchmarks, not to fuzzy rule-based systems (and due to the limited length of this paper). Optimal methods correlations with balance statistics together with the best fuzzy system solution (Table 4) show that fuzzy system-based methods outperform standard procedures in all cases except Hungary. (For Hungary, we have comparable results of correlation.) We find these results to be a promising premise for the application of fuzzy systems in the field of expectations quantification. 
Table 4 Summary of the best methods in the fuzzy and non-fuzzy approach

\begin{tabular}{llllll}
\hline Country & Optimal non-fuzzy methods & & \multicolumn{2}{l}{ Best fuzzy method } \\
\cline { 2 - 3 } & Name & R & & Name & R \\
\hline BG & CP_skewed_t-Student_obj_expectation_wav & 0.722 & & SBC_inf & $\mathbf{0 . 9 5 6}$ \\
CZ & CP_uniform_sub & 0.570 & & WM_inf2 & $\mathbf{0 . 7 8 0}$ \\
DK & CP_logistic_obj_inf & 0.343 & & SBC_inf2 & $\mathbf{0 . 8 7 0}$ \\
HR & CP_logistic_obj_expectation_wav & 0.823 & & SBC_inf2 & $\mathbf{0 . 9 2 6}$ \\
HU & CP_normal_sub & $\mathbf{0 . 9 2 4}$ & & SBC_inf & 0.921 \\
PL & CP_logistic_obj_expectation_wav & 0.790 & & SBC_inf2 & $\mathbf{0 . 9 7 4}$ \\
RO & CP_normal_obj_food & 0.680 & & SBC_inf & $\mathbf{0 . 9 8 5}$ \\
SE & CP_logistic_obj_inf & 0.745 & & SBC_inf2 & $\mathbf{0 . 8 4 3}$ \\
UK & CP_uniform_obj_inf & 0.559 & & SBC_inf & $\mathbf{0 . 9 2 2}$ \\
\hline
\end{tabular}

All results are statistically significant, bold shows the higher results. Significant level is 0.05

\subsection{Discussion}

As the results confirm, the fuzzy control approach is a promising method for estimating inflation expectations, and the best results are in places in which alternative methods are strongly needed. The key choice when creating a system is the choice of method for generating rules. WM methods had too small data sample to cover well partition, what have been reflected in the results, in particular when we would like to use more than 3 fuzzy sets describing each variable. SBC methods generated for the country on average from 2 (CZ, SE) to 9 (RO) clusters for each variables. It seems to us that optimisation of hyperparameters should improve them even further. As in any fuzzy modelling, knowledge of the problem was a critical factor, because the results are highly dependent on changes in the range of individual variables. Range 2 , which includes min and max as well as expert heuristics, displays better results.

A caveat regarding surveys' disadvantages should be also made. Literature discusses framing effect (Berk 1999; van der Klaauw et al. 2011), and a relation of surveys' responses to actual economic choices of responders (Manski 2004; Armantier et al. 2015). Fuzzy rule-based system application does not remove the objections towards surveys, what we have in mind while discussing our results.

\section{Conclusion}

In this paper, we present a novel application of fuzzy rulebased systems for detecting numerical values of consumers' expectations. Inflation expectations constitute a pivotal variable for central banks' policy conduct and are not directly observable. Thus, limitations regarding their approximation constrain their applicability for policy analysis. The algorithms applied in this study overcome the majority of objections that are presented towards standard methods of quantification. Except their obvious advantage - as there is no need to make assumptions about time series propertiesand their alignment to consumers' reduced economic literacy, fuzzy rule-based systems returned promising results when compared with standard quantification procedures. We also see room for further developments and applications of this novel application of fuzzy systems.

Funding Information This study was funded by Polish National Science Centre (the grant no. 2018/31/B/HS4/00164).

\section{Compliance with ethical standards}

Conflict of interest Authors declare that they have no conflict of interest.

Ethical approval This article does not contain any studies with human participants or animals performed by any of the authors. All authors contributed to the study conception and design. Material preparation and data collection were performed by Magdalena Szyszko. The analysis and code were done by Aleksandra Rutkowska. The first draft of the manuscript was written by Aleksandra Rutkowska, and Magdalena Szyszko commented on previous versions of the manuscript and made the interpretation of the results. All authors read and approved the final manuscript.

Open Access This article is licensed under a Creative Commons Attribution 4.0 International License, which permits use, sharing, adaptation, distribution and reproduction in any medium or format, as long as you give appropriate credit to the original author(s) and the source, provide a link to the Creative Commons licence, and indicate if changes were made. The images or other third party material in this article are included in the article's Creative Commons licence, unless indicated otherwise in a credit line to the material. If material is not included in the article's Creative Commons licence and your intended use is not permitted by statutory regulation or exceeds the permitted use, you will need to obtain permission directly from the copyright holder. To view a copy of this licence, visit http://creativecomm ons.org/licenses/by/4.0/. 
A Range of data set for fuzzy systems

\begin{tabular}{|c|c|c|c|c|c|c|c|}
\hline range & BG & & & & & & \\
\hline \multirow[t]{2}{*}{1} & Min & 5.04 & 16.14 & 3.56 & 0.68 & 0.00 & -3.54 \\
\hline & Max & 74.17 & 53.08 & 40.88 & 18.69 & 7.23 & 17.68 \\
\hline \multirow[t]{2}{*}{2} & Min & 0.00 & 10.00 & 0.00 & 0.00 & 0.00 & -5.76 \\
\hline & $\begin{array}{l}\text { Max } \\
\mathrm{CZ}\end{array}$ & 80.00 & 60.00 & 45.00 & 25.00 & 15.00 & 18.24 \\
\hline \multirow[t]{2}{*}{1} & Min & 1.35 & 5.93 & 2.88 & 5.90 & 0.33 & -0.23 \\
\hline & Max & 51.31 & 64.39 & 51.74 & 49.46 & 18.33 & 8.17 \\
\hline \multirow[t]{3}{*}{2} & Min & 0.00 & 0.00 & 0.00 & 0.00 & 0.00 & -2.00 \\
\hline & Max & 60.00 & 70.00 & 60.00 & 55.00 & 25.00 & 9.00 \\
\hline & HR & & & & & & \\
\hline \multirow[t]{2}{*}{1} & Min & 3.17 & 16.43 & 5.90 & 0.97 & 0.00 & -1.31 \\
\hline & Max & 72.18 & 53.79 & 33.40 & 41.42 & 6.93 & 8.54 \\
\hline \multirow[t]{3}{*}{2} & Min & 0.00 & 10.00 & 0.00 & 0.00 & 0.00 & -3.00 \\
\hline & Max & 80.00 & 60.00 & 40.00 & 50.00 & 10.00 & 8.00 \\
\hline & $\mathrm{HU}$ & & & & & & \\
\hline \multirow[t]{2}{*}{1} & Min & 2.64 & 15.93 & 4.85 & 0.20 & 0.00 & -1.84 \\
\hline & Max & 60.57 & 60.61 & 49.05 & 35.85 & 6.04 & 13.55 \\
\hline \multirow[t]{3}{*}{2} & Min & 0.00 & 10.00 & 0.00 & 0.00 & 0.00 & -4.00 \\
\hline & Max & 65.00 & 65.00 & 55.00 & 40.00 & 10.00 & 12.00 \\
\hline & DK & & & & & & \\
\hline \multirow[t]{2}{*}{1} & Min & 1.00 & 4.40 & 5.00 & 3.97 & 0.11 & -0.25 \\
\hline & Max & 35.66 & 56.60 & 46.27 & 59.80 & 26.49 & 5.26 \\
\hline \multirow[t]{3}{*}{2} & Min & -5.00 & 0.00 & 0.00 & 0.00 & -5.00 & -1.00 \\
\hline & Max & 40.00 & 60.00 & 50.00 & 65.00 & 35.00 & 6.00 \\
\hline & PL & & & & & & \\
\hline \multirow[t]{2}{*}{1} & Min & 0.70 & 15.20 & 4.00 & 2.70 & 0.00 & -0.80 \\
\hline & Max & 49.00 & 62.00 & 44.80 & 44.30 & 5.00 & 9.10 \\
\hline \multirow[t]{3}{*}{2} & Min & 0.00 & 10.00 & 0.00 & 0.00 & 0.00 & -4.00 \\
\hline & Max & 55.00 & 70.00 & 50.00 & 50.00 & 10.00 & 7.00 \\
\hline & $\mathrm{RO}$ & & & & & & \\
\hline \multirow[t]{2}{*}{1} & Min & 2.50 & 8.74 & 4.47 & 1.00 & 0.00 & -5.59 \\
\hline & Max & 67.89 & 51.46 & 39.33 & 51.30 & 23.24 & 30.01 \\
\hline \multirow[t]{3}{*}{2} & Min & 0.00 & 0.00 & 0.00 & 0.00 & 0.00 & -10.00 \\
\hline & Max & 75.00 & 60.00 & 45.00 & 55.00 & 30.00 & 35.00 \\
\hline & SE & & & & & & \\
\hline \multirow[t]{2}{*}{1} & Min & 0.30 & 2.00 & 1.00 & 8.10 & 0.20 & -0.41 \\
\hline & Max & 49.80 & 44.20 & 58.50 & 75.60 & 21.80 & 6.83 \\
\hline \multirow[t]{3}{*}{2} & Min & 0.00 & 0.00 & 0.00 & 0.00 & 0.00 & -4.00 \\
\hline & $\operatorname{Max}$ & 55.00 & 50.00 & 65.00 & 80.00 & 25.00 & 6.00 \\
\hline & UK & & & & & & \\
\hline \multirow[t]{2}{*}{1} & Min & 3.90 & 13.60 & 7.12 & 5.60 & 0.78 & -0.25 \\
\hline & $\operatorname{Max}$ & 56.56 & 43.64 & 41.37 & 37.80 & 18.08 & 6.49 \\
\hline \multirow[t]{2}{*}{2} & Min & 0.00 & 5.00 & 0.00 & 0.00 & 0.00 & -2.00 \\
\hline & Max & 65.00 & 50.00 & 50.00 & 45.00 & 25.00 & 7.00 \\
\hline
\end{tabular}

B Correlation results of benchmark methods

\begin{tabular}{lll}
\hline & $C P_{\text {sub }}$ & $C P_{\text {obj_inf }}$ \\
\hline BG & 0.64 & 0.69 \\
CZ & 0.66 & 0.31 \\
DK & 0.42 & 0.33 \\
HR & - & 0.73 \\
HU & 0.92 & 0.81 \\
PL & 0.78 & 0.75 \\
RO & 0.54 & 0.64 \\
SE & 0.55 & 0.72 \\
UK & 0.32 & 0.54 \\
\hline
\end{tabular}

\section{References}

Acedański J, Włodarczyk J (2016) Dispersion of inflation expectations in the european union during the global financial crisis equilibrium. Q J Econ Econ Policy 11(4):737-749

Anderson O (1952) The business test of the IFO-institute for economic research, munich, and its theoretical model. Rev Int Stat Inst 20(1):1-17

Armantier O, Bruine de Bruin W, Topa G, van der Klaauw W, Zafar B (2015) Inflation expectations and behavior: do survey respondents act on their beliefs? Int Econ Rev 56(2):505-536

Batchelor RA, Orr AB (1988) Inflation expectations revisited. Economica 55(219):317

Berk JM (1999) Measuring inflation expectations: a survey data approach. Appl Econ

Carlson JA, Parkin M (1975) Inflation expectations. Economica 42(166): $123-38$

Chiu S (1996) Method and software for extracting fuzzy classification rules by subtractive clustering. In: Proceedings of North American Fuzzy Information Processing, pp 461-465. IEEE

Czech National Bank (2007) Inflation Report 2007Q3

European Commision (2020) The Joint Harmonised EU Programme of Business and Consumer Surveys. User Guide

Henzel S, Wollmershäuser T (2005) An alternative to the CarlsonParkin method for the quantification of qualitative inflation expectations: evidence from the ifo world economic survey

Lahiri K, Zhao Y (2015) Quantifying survey expectations: a critical review and generalization of the Carlson-Parkin method. Int J Forecast 31(1):51-62

Lolić I, Sorić P (2018) A critical re-examination of the Carlson-Parkin method. Appl Econ Lett 25(19):1360-1363

Mamdani E, Assilian S (1975) An experiment in linguistic synthesis with a fuzzy logic controller. Int J Man-Mach Stud 7(1):1-13

Manski CF (2004) Measuring expectations. Econometrica 72(5):1329_ 1376 
Muth JF (1961) Rational expectations and the theory of price movements. Econometrica 29(3):315

Nguyen A-T, Taniguchi T, Eciolaza L, Campos V, Palhares R, Sugeno M (2019) Fuzzy control systems: past, present and future. IEEE Comput Intell Mag 14(1):56-68

Pesaran M (1984) Expectations formation and macroeconometric modelling. In: Muet P-A (ed) Contemporary macroeconomic modelling. Basil Blackwell, Oxford

Pesaran M, Weale M (2006) Survey expectations. In: Handbook of economic forecasting

Simmons P, Weiserbs D (1992) Consumer price perceptions and expectations. Oxford Econ Pap 44(1):35-50

Smith J, McAleer M (1995) Alternative procedures for converting qualitative response data to quantitative expectations: an application to Australian manufacturing. J Appl Econom

Theil H (1952) On the time shape of economic microvariables and the munich business test. Rev Int Stat Inst 20:105-120 van der Klaauw W, Bruine de Bruin W, Topa G, Potter S, Bryan MF (2011) Rethinking the measurement of household inflation expectations: preliminary findings. SSRN Electron J

Wang L-X, Mendel JM (1992) Generating fuzzy rules by learning from examples. IEEE Trans Syst Man Cybern 22(6):1414-1427

Yager RR, Filev DP (1994) Generation of fuzzy rules by mountain clustering. J Intell Fuzzy Syst 2(3):209-219

Zadeh LA (1973) Outline of a new approach to the analysis of complex systems and decision processes. IEEE Trans Syst Man Cybern $1: 28-44$

Zadeh LA (1996) On fuzzy algorithms. In: Fuzzy sets, fuzzy logic, and fuzzy systems: selected papers By Lotfi A Zadeh, pp 127-147. World Scientific

Publisher's Note Springer Nature remains neutral with regard to jurisdictional claims in published maps and institutional affiliations. 